\title{
Fiber-optical sensor with intensity compensation model in college teaching of physics experiment
}

Liping Su, Yang Zhang, Kun Li, Yu Zhang

Liping Su, Yang Zhang Sr., Kun Li Sr., Yu Zhang, "Fiber-optical sensor with intensity compensation model in college teaching of physics experiment," Proc. SPIE 10452, 14th Conference on Education and Training in Optics and Photonics: ETOP 2017, 1045265 (16 August 2017); doi: 10.1117/12.2269912

SPIE Event: 14th Conference on Education and Training in Optics and Photonics, ETOP 2017, 2017, Hangzhou, China 


\title{
Fiber-optical Sensor with Intensity Compensation Model in College Teaching of Physics Experiment
}

\author{
Liping Su, Yang Zhang*, Kun Li, Yu Zhang
}

Key Laboratory of integrated fiber optics, Ministry of education. National Demonstration Center for Physical Experiment Education, Harbin Engineering University, China

\begin{abstract}
Optical fiber sensor technology is one of the main contents of modern information technology, which has a very important position in modern science and technology. Fiber optic sensor experiment can improve students' enthusiasm and broaden their horizons in college physics experiment. In this paper the main structure and working principle of fiberoptical sensor with intensity compensation model are introduced. And thus fiber-optical sensor with intensity compensation model is applied to measure micro displacement of Young's modulus measurement experiment and metal linear expansion coefficient measurement experiment in the college physics experiment. Results indicate that the measurement accuracy of micro displacement is higher than that of the traditional methods using fiber-optical sensor with intensity compensation model. Meanwhile this measurement method makes the students understand on the optical fiber, sensor and nature of micro displacement measurement method and makes each experiment strengthen relationship and compatibility, which provides a new idea for the reform of experimental teaching.
\end{abstract}

Keywords: intensity compensation, optical fiber sensor, college physics experiment, micro displacement *Second Author Email: zhangyang@hrbeu.edu.cn

\section{INTRODUCTION}

With development of optical fiber technology, optical fiber sensor is more and more extensively applied in the measurement field. Optical fiber displacement sensor with intensity compensation model has its unique advantages in measurement. It not only has the advantages of simple structure, flexible design, reliable performance and low cost, but also is easy to realize the measurement of various physical quantities, such as stress, vibration, temperature and degree of surface roughness ${ }^{[1]}$.

The development of modern science and technology has brought new vitality to physics experiment, and the integration of modern technology and experiment teaching is the inevitable trend of the development of physics curriculum. By adding optical fiber sensing experiment, the new modern optical technology is widely used and popularized, which not only enriches the teaching content of college physics experiment, but also broadens the knowledge of students. In turn, students study college physics with the more enthusiasm, and are guided to enter the field of engineering applications.

\section{BRIEF INTRODUCTION OF FIBER OPTIC DISPLACEMENT SENSOR WITH INTENSITY COMPENSATING MODE}

The optical fiber displacement sensor with intensity compensation mode is shown in Figure 1 [2]. It is mainly composed of digital voltmeter of optical fiber sensing experiment instrument, a three optical fiber sensing probe with compensation and a lens adjusting frame. The optical fiber probe can be controlled by the screw micrometer to displace up and down. Its resolution is up to $0.001 \mathrm{~mm}$, and the precision of the digital voltmeter of is $0.1 \mathrm{mV}$.

14th Conference on Education and Training in Optics and Photonics: ETOP 2017, edited by Xu Liu,

Xi-Cheng Zhang, Proc. of SPIE Vol. 10452, 1045265 • ( 2017 ICO, IEEE, OSA, SPIE

CCC code: $0277-786 X / 17 / \$ 18 \cdot$ doi: $10.1117 / 12.2269912$ 


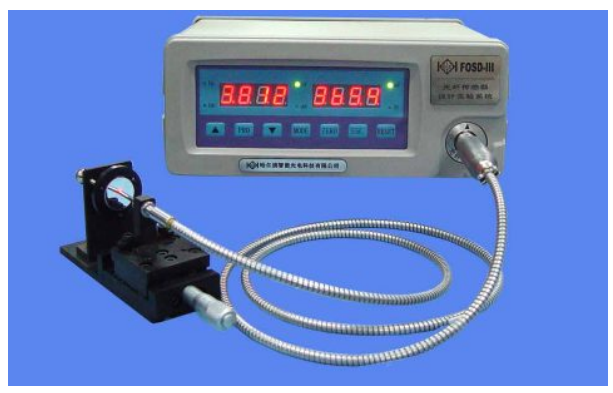

Figure 1 The fibre-optical sensor with intensity compensation model

Figure 2 is the physical diagram and schematic diagram of the three optical fiber sensing probe with compensation[2]. The sensing probe consists of one incident optical fibers and two receiving optical fibers. Incident optical fiber and receiving optical fiber are the same kind of optical fiber and three fibers form an optical cable. The light emitted by the light source LED radiates onto the reflecting mirror by the incident optical fiber, the light by reflected back by the mirror is received by the two receiving optical fibers, and then radiates to the photoelectric detector PIN. The photoelectric detector converts the optical signal into the electrical signal, which is processed and amplified through the filter amplifier circuit. The received received light intensity depends on the distance between the mirror and the optical fiber probe. When the distance between the mirror and the optical fiber probe changes, the output light intensity of the receiving optical fiber will change accordingly, therefore the relative micro displacement of the mirror or the optical fiber probe can be measured by detecting the output light intensity.

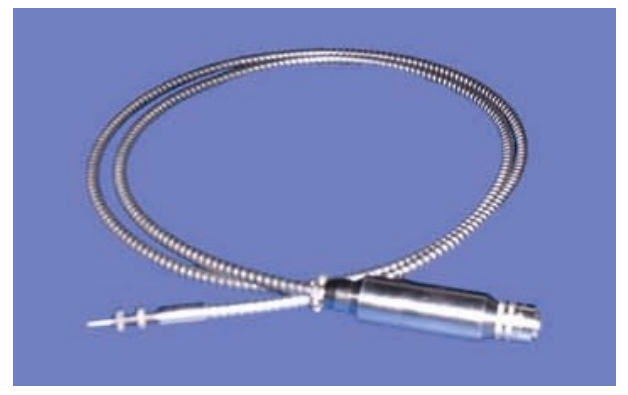

(a) the physical map

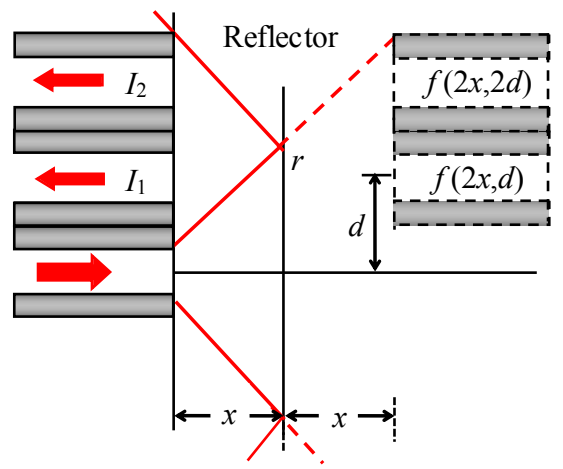

(b) the schematic diagram

Figure 2 the physical map and schematic diagram of the three optical fiber sensing probe with compensation

The two light intensities received respectively by the two receiving optical fibers is from the same source and the same reflective object, therefore they can compensate the influences on the received intensities of light source intensity fluctuation and surface reflectivity changes. Moreover, the incident optical fiber and receiving optical fiber are the same 
kind of optical fiber and three fibers form an optical cable, therefore they can compensate for influences on the received intensities of optical fiber bending, environmental temperature and pressure etc. Thus, we can eliminate those influences of these interference factors on the measurement results by the ratio operation, that is to say, the ratio of the output light intensities of the two receiving fibers is taken as the characteristic modulation function of the optical fiber sensor [3]:

$$
I_{1} / I_{2}=\exp \left\{-\frac{d_{1}^{2}-d_{2}^{2}}{\omega^{2}(2 x)}\right\}
$$

From the above formula we can see that as long as $d^{1}$ and $d^{2}$ (fiber spacing) are not equal, which makes the coupling conditions of three optical fibers not to change, output intensity ratio of the two receiving optical fibers is only relate to the distance between the optical fiber end and the reflective mirror, and not to the intensity of the light source, the mirror reflectivity, fiber transmission loss and other interference factors. Namely, the optical fiber sensing system with intensity compensation mode can automatically compensate for influences of environmental interference factors.

\section{APPLICATION OF FIBER OPTIC SENSOR WITH COMPENSATION IN COLLEGE PHYSICS EXPERIMENT}

\subsection{Application in the measurement of Young's modulus}

Young's modulus is an important physical quantity which reflects the ability of resisting elastic deformation of the materials. Young's modulus is usually used as one of the basis of selecting materials in Engineering. There are many methods of measurement of Young's modulus. Usually the static stretching—optical lever amplification method is used in college physics experiment. Due to the micro elongation of wire stretching, there are adjustment difficulties, poor stability, large error and low technology level and Young's modulus is not easy to be measured in the actual experiment. The measurement accuracy can only be $10^{-2} \mathrm{~m}$ of the order of magnitude. While the use of the optical fiber sensor with intensity compensation to measure the micro elongation, the measurement accuracy can be $10^{-7} \mathrm{~m}$ of the order of magnitude. The measuring device is shown in Figure $3^{[4]}$. The reflective mirror is fixed on the lower surface of the lower chuck, and then the optical fiber probe is fixed at a position below the reflective mirror.

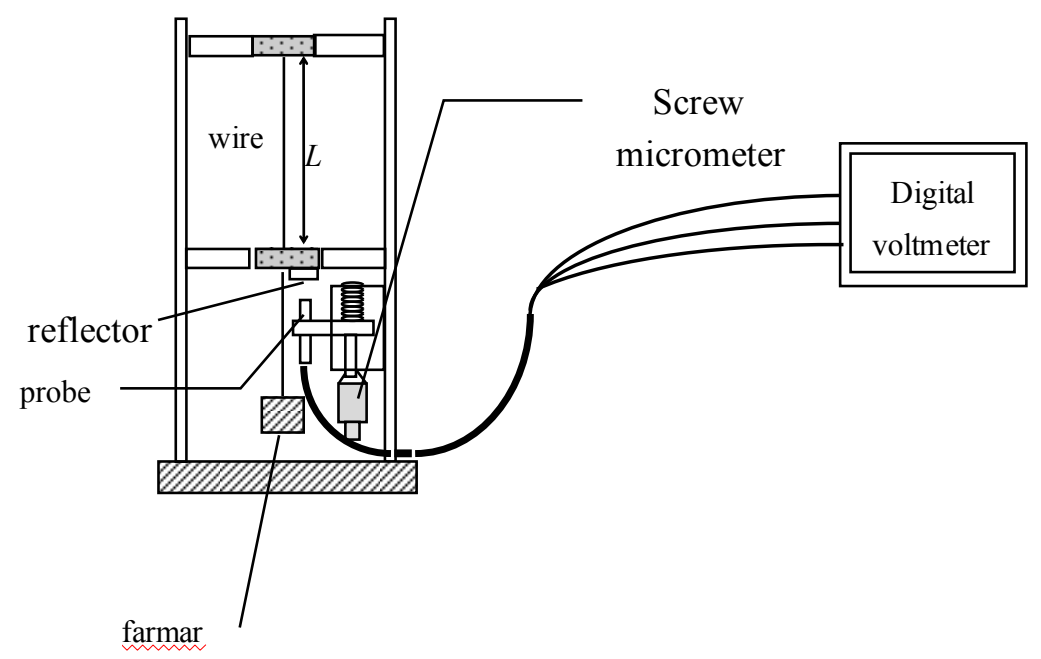

Figure 3 Schematic diagram of measuring Young's modulus by optical fiber sensor

Because the output of the optical fiber experiment instrument is the voltage value and the displacement change is needed, the relationship between the output voltage and the displacement change is obtained before the measurement. The screw micrometer is used to calibrate the optical fiber sensor. The three optical fiber sensing probe is justly contact with the 
reflective mirror, the screw micrometer is adjusted, and then the optical fiber probe is slowly away from the reflective mirror. In this process, output voltage value of the experimental instrument is recorded at every fixed distance. The characteristic curve of the optical fiber sensor is made to reflect relationship between the distance of the probe and mirror and the output voltage. On this characteristic curve the linear part is used as the calibration curve of the optical fiber sensor. Then the micro displacement is measured by increasing (decreasing) the farmar weight per time.

The optical fiber sensor is introduced to measure the micro displacement, so the experiment precision is improved, the knowledge of science and engineering students is expanded, and heir practical ability is improved.

\subsection{Application in the measurement of solid linear expansion coefficient}

Thermal expansion and cold contraction is an important physical phenomenon. In the one-dimensional case, the increase in the length of the heated solid is called to be linear expansion. Under the same conditions, the linear expansion of solid is different due to the different materials. Therefore linear expansion coefficient is used to represent the characterization of the solid linear expansion. The linear expansion coefficient is one of the basic physical parameters of materials. Although the thermal expansion is not very large, it can produce great stress. So it is important to measure the linear expansion coefficient of solid in engineering correctly. The key to measurement method of linear expansion coefficient is to measure accurately the length of the measured metal at a series of temperatures, and then calculate the linear expansion coefficient according to the temperature difference and the length difference between two adjacent temperatures. Various types of linear dilatometers are used to determinate the linear expansion coefficient. They are mainly composed of two parts: temperature control system and micro displacement measurement system. The main problem is how to measure the micro displacement for measuring the linear expansion coefficient. The micro displacement can be measured mot only by the micrometer, but also the optical lever amplification and optical interference method.

Device for measuring the metallic linear expansion coefficient is shown in Figure 4 by using the optical fiber sensor with intensity compensation ${ }^{[3]}$. In the figure the measured metal rod can be placed in the glass tube with the heated water (fixed by the bracket P2 and P3), H1 is the inlet and H2 is the outlet. One end of the metal rod is fastened on the support of $\mathrm{P} 1$, the other end can stretch freely after heating. The thermometer $\mathrm{T}$ is inserted from the $\mathrm{C}$ hole and contact the metal rod, thus can measure the temperature of the metal rod. In Figure 4, R is the reflective mirror connected with the stretched rod, $\mathrm{O}$ is the optical fiber probe which can freely slide on the support of $\mathrm{P} 4$, and $\mathrm{M}$ is the spiral micrometer connected with the optical fiber probe, which is used for the calibration of the optical fiber sensor with intensity compensation. After calibration the optical fiber probe is fixed. In this way, the small elongation of the metal rod with temperature is converted to the micro displacement of the mirror.

By measuring the micro displacement by using the optical fiber sensor with intensity compensation mode, there is high sensitivity, good stability, strong anti-interference and reliable work in the solid linear expansion coefficient experiment. At the same time, no electric testing is achieved. Because modern optical fiber sensor technology is applied for the traditional physical experiment, which expands the application of optical fiber sensor in physics experiment.

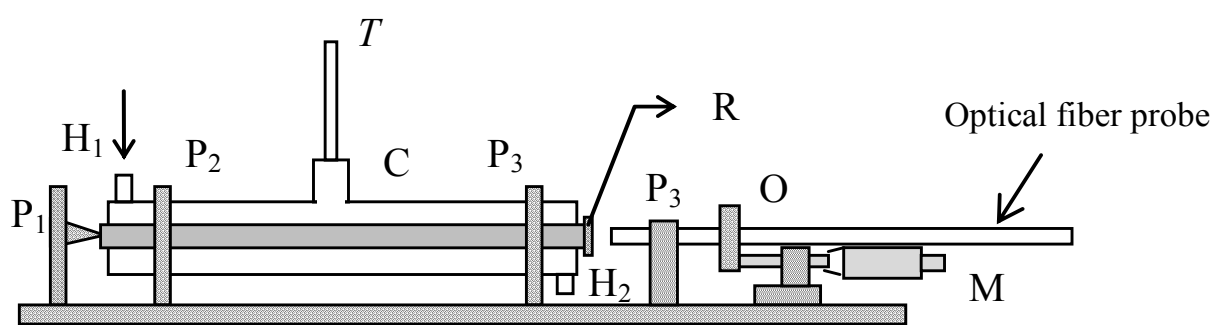

Figure 4 Schematic diagram of measuring metal linear expansion coefficient by optical fiber sensor 


\section{CONCLUSION}

In this paper, the basic structure and working principle of the fiber-optical sensor with intensity compensation mode are introduced, and the micro displacement in Young's modulus and metal linear expansion coefficient measurement experiment is measured by using this sensor. With combination of the optical fiber sensor technology and college physics experiment, students not only master the principle of optical fiber sensor experiment and fully understand the output characteristics of the optical fiber sensor with intensity compensation, but also know influence factors of its output characteristic curve. At the same time students can expand their knowledge, improve their abilities for analyzing problems and solving problems, stimulate their interest in learning and cultivate their study ability.

\section{REFERENCES}

[1] Shen, F. And Wang, P., "Research the output characteristics of fiber-optic displacement sensor in the experimental teaching," China Modern Educational Equipment23,114-118(2011)

[2] Sun, J.H.,Wang, X.F. And Chen, S.Y., “University physics experiment course,” Harbin:Harbin engineering university press, 2016

[3] Jia, Y.M., Yang, S.K. and Zhu, J., "Measuring linear expansion coefficient of metal using optical fiber sensor with intensity compensation," Physics Experimentation28(10),1-4(2008)

[4] YE, T. M., Zhou, Y. D., Pan, N., "Measuring Young modulus of wire with optical fiber sensor," Physics Experimentation35(12),36-38(2015) 